\title{
Design of Embedded Numerical Control System Based on ARM
}

\author{
Dongdong Li, Zhiqin Wei \\ School of Mechanical Engineering Guangzhou College of South China University of Technology \\ *lidd@gcu.edu.cn
}

Keywords: ARM; Embedded system; Numerical Control

\begin{abstract}
The emergence of embedded technology brought a new technological revolution for the field of modern industrial numerical control. Based on the analysis of embedded technology and computer numerical control systems, this paper discussed the general structure model of embedded numerical control system, analyzed the design method of hardware and software, including design of basic drive module, multi-task in parallel of the system, and so on.
\end{abstract}

\section{Introduction}

Embedded CNC(Computer Numerical Control) system has become an important trend of the development of CNC system. The use of embedded system technology in CNC system not only makes the abundance of CNC system hardware and software resources, but also improves the reliability of the whole CNC, taking advantage of the specificity of embedded system's software and hardware, as well as its customizability. Embedded CNC combines embedded system technology and traditional CNC technology, with the characteristics of strong function, low cost, small size and so on. In terms of social benefits, embedded $\mathrm{CNC}$ can greatly popularize numerical control with industrial prospect [1].

Embedded systems provides a flexible and convenient control system for numerical control technology, which can be embedded in the industrial system, capable of continuous long-term reliable operation in the industrial environment for micro and cheap control system. At present, the research and application of embedded systems, has become a new trend.

ARM (Advanced RISC Machines) processor is a high performance and low power embedded microprocessor. Transplanting the real-time operating system on the ARM processor can improve the reliability and stability of the system, realize the real-time management and improve the performance of the system.

This CNC system uses ARM processor, developing numerical device of high integration, stability, high speed and accuracy.

\section{Analysis of Embedded CNC and its General Structural Model Design}

$\mathrm{CNC}$ system receives code information from input device, and various information and instructions are output after compilation, arithmetic and logic processing by logic circuit or system software of NC, and controls all parts of the machine, provides orderly movements[2].

Design of embedded system generally consists of two parts: design of hardware platform and software design. Design of hardware platform includes choice of embedded processors, peripheral circuit design and selection of development tool hardware is also crucial. Then we can do the choice of OS (Operating System) and the development of application. The choice of OS is the key to the entire software design; it relates to the difficulty of system development, system operation stability and reliability, and application development, and so on. Before the operating system starts, system initialization is implemented, initializing the system execution environment, basic equipment. Then the transplant of the operating system, the driver development and finally the application development and testing on the operating system are implemented. 
The embedded numerical control system is composed of three layers--the hardware layer, the operating system layer and the numerical control software layer. The hardware layer of the embedded NC system is composed of the hardware structure of the ARM processor and the motion control chip.

As the first step in the research of Embedded NC system, S3C44B0X processor development board is used, which integrates the ARM processor S3C44B0X, memory, FLASH, ARM memory and so on.

The development board also integrates the functions of network and serial port, power and manual reset, which greatly reduces the workload in hardware development. ARM development board and the external circuit, constitute the ARM processor hardware architecture.

Operating system layer of embedded CNC system uses Linux, which is an open source code embedded real-time operating system. Linux is a multitasking operating system, which has a feature of being able to be cut according to the needs.

Using Linux makes control software of embedded CNC system relatively simple. So the system has the ability of multi task processing and good real-time performance. The operating system layer includes memory management, task management, device management, motion control chip interface, communication interface, etc. In the top is the numerical control system matching software, mainly including the various control functions, such as control function of motion control chip, etc.

\section{Hardware Design of Embedded CNC}

A master-slave dual CPU structure model is used in the embedded numerical control system. The main CPU is ARM processor, which is used in the management of keyboard input, LCD, serial port and network communication, and to control the slave CPU through read and write bus, which is motion control chip, PCL6045, used to complete the complex motion control. The communication between PCL6045 and ARM processor is to read and write several addresses on the bus to carry out instructions and data transmission. In addition, the system's input and output I/O port, keyboard, network and so on is also controlled by the bus. Figure 1 shows the overall structure of the hardware of the embedded CNC system.

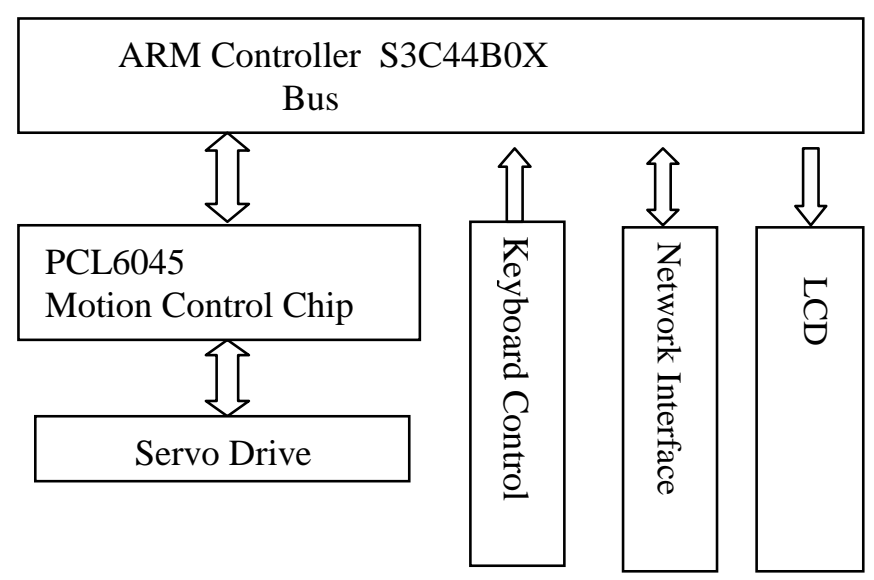

Fig.1 General Structure of Hardware in Embedded CNC

Connection Between PCL6045 and 3C44B0X PCL6045 is a powerful DSP motion control chip in PCL series. PCL/PCD series chip is a special purpose DSP (digital signal processing) motion processor designed for the purpose of stepping and servo control. With its features of powerful, simple and easy to use, excellent quality, and competitive prices, PCL6045 has occupied most of the market in the United States, Europe and Japan, and become the world's first brand. PCL6045 can receive commands from the bus interface or support manual input for real-time motion control, including uniform and variable speed pulse transmission, lifting speed planning, linear or circular interpolation, etc. The highest frequency of the chip output pulse reaches $6.5 \mathrm{MHZ}$. The chip can 
control four axes interpolation and perform continuous interpolation motion of multi segment line and circular arc.

In order to simplify the structure of the software, a function library is built, which is used to manage the operation of the PCL6045 chip. According to the data book of PCL6045 chip and the specific use of the system, the function library includes dozens of functions, such as:

1) Parameters setting functions (setting distance, velocity, acceleration, etc.).

2) State reading functions (reading the current position, origin and limit switch state, etc.).

3) Motion functions (point position motion, continuous motion, circular interpolation, return to zero, etc.).

4) Stop and interrupt management functions (rush and deceleration, etc.).

The control function library of PCL6045 motion control chip is an important part of the software system.S3C44B0X's address and data line separation, which has RD, WR, WAIT read and write control signal line.

Design of the Basic Drive Module of the System According to the design of system hardware, the basic drive module includes: storage unit, display unit, keyboard unit, I/O unit, D/A unit, A/D unit, serial communication and network communication [3]. Below are the function, design principles and the main interface of the drivers.

Storage unit driver: the driver module encapsulates the RAM area, FLASH read / write / removal and other low-level data operations, whose external interface format is divided by byte, word, double word, data block, which is the upper data management module.

Display drive unit: display unit driving encapsulates the basic operation of the LCD module, using the basic instruction SED1335 controller provides, provides the basic call interface for display of text, graphics, etc., and encapsulates some commonly used graphic structure in the form of control for the upper software.

Keyboard unit driver: the driver module encapsulates the keyboard scan subroutine, and can be invoked by timing routine, by specifying the scan time, the time for eliminating the jitter and providing keyboard encoding table and other parameters.

I/O unit driver: input and output driver module, which provides reset operation for some independent I/O interface, and is encapsulated as reading and writing call in the form of control word.

Serial communication driver: implements the basic operating functions, such as low-speed data stream transmitting, receiving, transmission, error detection and encryption.

Network communication driver: the network card driver implements the protocol of TCP/IP, and establishes a complete network connection and transmission framework.

\section{Software Design of Embedded CNC}

Embedded CNC software system takes embedded operating system as the core, through its core which is responsible for dynamic management system memory, implements task scheduling, coordinates the communication between tasks using signal, mailbox mechanism. It also implements the communication between tasks, procedures through message queue[4].

The system software can be divided into five modules: system boot program Bootloader, embedded operating system kernel, device driver, API(Application Programming Interface) function, system task and application program.

Bootloader is a program that runs before the operating system kernel. Through this program, you can initialize the hardware device, set up the boot parameter area, build the mapping graph of the memory space, construct the parameter structure and identify list (the kernel needs to use the boot parameter to identify the root device, page size, memory size), so as to make the system's software and hardware environment in a suitable state.

Embedded operating system implements the core functions, such as task scheduling and control of embedded applications. It has the characteristics of compact core, configurable, and closely related with high level application. 
Device driver provides direct control and the use of the underlying hardware resources, and as a link between the underlying hardware and the upper API function, separating the underlying hardware and API functions, so that the underlying hardware changes will not affect API functions and applications. Therefore it is easy to maintain and upgrade the system only by changing the corresponding hardware drivers.

API function provides the user with the basic control of the development and management system interface. Users can directly call the corresponding API functions for the application development based on the actual needs. In this way, not only the complexity of the application development is reduced and the development speeds up, but also the portability of applications is ensured and the convenience for upgrade is realized. [5]

The function of system task module is to coordinate the scheduling between the main tasks to ensure the efficiency and real-time performance of the task.

A series of specific functions can be realized through the application program. Users write the application according to the actual needs by calling the corresponding API function, the operation of the controlled object, to achieve the required functions. The coordination between application tasks can be implemented with the help of the system's message queue mechanism. [6]

\section{Debugging and Conclusions}

System debugging is carried out in the quilting CNC machine, which includes a main control box with a motor, a tachometer disc and various other sensors.

Embedded CNC system is a combination of embedded system and numerical control technology, which has become an important direction in the development of numerical control system. The design develops an embedded CNC system with dual CUP, ARM and DSP. The main CPU is based on the ARM core S3C44B0X. And the slave CPU is PCL6045 motion control chip, which is used to complete the complex motion control. In the software, the free open source Linux system is used as the software platform, and the Linux is improved to meet the real-time requirements of industrial control field. On this basis, the main functional modules of the CNC system were analyzed and designed.

\section{Acknowledgment}

The authors would like to thank the Outstanding Young Backbone Teachers Project supported by Guangzhou College of South China University of Technology.

\section{References}

[1] Wang Hujun, "Research on the development of domestic and international numerical control system", Science Education, vol 2, 2011, p. 179

[2] Xu Yizhen, "Embedded CNC milling system research based on ARM and real-time Linux", Nanjing Aerospace University, 2012, pp.50-55.

[3] Samsung Electronics S3C44B0X USER' S MANUAL Revision 1.Korea, Samsung Electronics Co. ,Ltd.

[4] Zhou Junjie, He Panfeng, "Design of the real-time multi task software framework based on VxWorks", Foreign electronic measurement technology, vol. 31(4), 2012, pp. 80-82.

[5] He Haibo, "Study on key technologies of intelligent home furnishing system based on embedded Linux", Anhui Institute of Technology, 2012.

[6] Cao Yuhua, Yu Youpeng, "Research on real-time embedded CNC system based on Xenomai", Manufacturing Technology and Machine, 2011, (6), pp. 51 55. 